\title{
Prognostic Relevance of Mitotic Activity in Patients with Node-Negative Breast Cancer
}

Laura Medri, B.Sc., Annalisa Volpi, B.Sc., Oriana Nanni, M.Sc., Anna Maria Vecci, B.Sc., Annita Mangia, B.Sc., Francesco Schittulli, M.D., Franco Padovani, M.D., Donata Casadei Giunchi, M.D., Alfredo Vito, M.D., Dino Amadori, M.D., Angelo Paradiso, M.D., Rosella Silvestrini, Ph.D.

Pathology Unit (LM, FP) and Department of Medical Oncology (AnV, DCG, DA), Division of Oncology and Diagnostics, Pierantoni Hospital, Forlì; Istituto Oncologico Romagnolo (ON, AMV, RS), Forlì; and Clinical Experimental Oncology Laboratory (AM, AP), Breast Unit (FS), and Histopathology Service (AlV), Oncology Institute, Bari, Italy

The prognostic relevance of mitotic activity was analyzed in a series of 306 patients with node-negative breast cancer treated with locoregional therapy alone, until early relapse. Mitotic activity was evaluated as the number of mitotic figures per 10 highpower fields (mitotic activity index) or per 1000 tumor cells (mitotic index). Counting was carried out blindly by two observers. A high correlation was observed between the two determinations $\left(r_{\mathrm{s}}=.96\right.$, $P<.001)$. For clinical analysis, three mitotic activity index subgroups (mitotic figures/field $\leq$ 9, 10-19 and more than 19, according to grading criteria) and three mitotic index subgroups (percentage of mitotic figures less than $0.10,0.11-0.50$ and more than 0.50 , according to tertile criteria) were considered. No relation was observed between mitotic variables and 6-year disease-free survival, whereas distant disease-free survival was strongly related to mitotic figures per 10 fields $(85 \%, 89 \%$ and $70 \%, P=$ .012 ) and to the percentage of mitotic figures out of a total 1000 tumor cells $(87 \%, 86 \%$ and $75 \%, P=$ .017). Similarly, both mitotic indices were significantly related to 6 -year overall survival $(99 \%, 95 \%$ and $77 \%, P<.001$, for mitotic figures per 10 fields and $99 \%, 93 \%$ and $82 \%, P<.001$, for the percentage of mitotic figures). These findings were particularly evident in patients with tumors of $1-2 \mathrm{~cm}$. In conclusion, a high number of mitotic figures is associated with a higher probability of developing distant metastases and a shorter survival. The critical point

Copyright $\odot 2003$ by The United States and Canadian Academy of Pathology, Inc.

VOL. 16, NO. 11, P. 1067, 2003 Printed in the U.S.A.

Date of acceptance: July 21, 2003.

Supported by Istituto Oncologico Romagnolo, Forlì and by the National Research Council (CNR-Progetto Strategico MIUR), Grants 02.00452.ST97 and 02.00105.ST97, Rome, Italy.

Address reprint requests to: Laura Medri, B.Sc., Pathology Unit, Division of Oncology and Diagnostics, Pierantoni Hospital, via Forlanini 34, 47100 Forlì, Italy; fax: 390543 731736; e-mail: res.onco@ausl.fo.it.

DOI: 10.1097/01.MP.0000093625.20366.9D remains the standardization of the preanalytical and analytical steps within quality control programs.

KEY WORDS: Mitotic activity index, Mitotic index, Node-negative breast cancer, Prognosis.

Mod Pathol 2003;16(11):1067-1075

In the era of biomolecular markers, the everincreasing number of factors involved in breast cancer development and progression has overwhelmed researchers because of the complexity of the mechanisms underlying tumor cell growth.

It has been demonstrated that very many oncogenes, oncosuppressors, enzymes and growth factors, or hormone receptors play a role in specific stages of different tumor types. Interest in the prognostic and predictive clinical relevance of biological variables has focused on a relatively small number of factors, and the often conflicting results have puzzled clinicians with regard to their clinical use.

Among the different markers, cell proliferation can reasonably be supposed to be related to tumor aggressiveness, as it does not measure a causal event influenced by the complex interrelations of cell regulation but rather represents an epiphenomenon. Proliferative activity can be determined using various methods based on different rationales: evaluation of cells during DNA synthesis through the incorporation of specific precursors such as thymidine or bromodeoxyuridine, or flow cytometric DNA content and growth fraction through the expression of proliferation-associated antigens such as Ki67/MIB-1 or PCNA.

Recently, a renewed interest was witnessed in one of the proliferation markers first used, the mitotic index (MI), which is the fraction of cells in mitosis at any given time.

For all these methodologies, tissue fixation, staining or labeling techniques, scoring systems, and 
determination criteria may affect the results. In breast cancer, among the above-mentioned proliferation markers, only thymidine labeling index (TLI) has successfully undergone all the technical and clinical validation steps. In particular, it has been analyzed in large and homogeneous case series of patients with untreated node-negative breast cancer to define its prognostic relevance $(1,2)$ and in prospectively planned adjuvant chemotherapy trials to determine its predictive role in the response to systemic treatment $(3,4)$. Moreover, its clinical relevance has been assessed in both univariate and multivariate analyses and biological determinations have always been conducted within intra- and interlaboratory quality control programs.

Mitotic activity is currently used mainly as part of the tumor grading system. To our knowledge, few studies have investigated its relevance in large series of breast cancer patients with negative axillary nodes (5-8). However, a part of the patient populations studied was treated with systemic therapy, making it impossible to evaluate the pure prognostic value of this variable. Moreover, unlike other proliferation markers, there are insufficient data on the value of mitotic activity as a predictor of response to systemic anticancer therapies.

The aim of the present study was to evaluate the pure prognostic value of mitotic activity in an adequate series of patients with node-negative breast cancer treated with locoregional therapy alone, as a requisite for defining and then prospectively using the variable to identify patients at risk. The present study was conducted under strict intra- and interlaboratory quality control conditions for mitotic activity determination and with close patient follow up.

\section{MATERIALS AND METHODS}

The clinicobiological study was conducted on a series of 306 patients with histologically confirmed node-negative (at least 10 axillary lymph nodes examined) breast cancer radically resected and recruited between 1989 and 1993 by the Oncology Institutes of Romagna (IOR) and Bari (IRCCS Bari). All patients received locoregional therapy alone (surgery \pm radiotherapy): $>50 \%$ underwent quadrantectomy plus radiotherapy and the remaining patients were subjected to mastectomy. No adjuvant systemic treatment was given until early relapse.

Patient distribution according to different clinicopathological and biological characteristics is reported in Table 1 . Median age of patients was 55 years (range, 29-74 y) and $>60 \%$ of patients were postmenopausal. About two thirds of tumors were $\leq 2 \mathrm{~cm}$ in diameter. Considering the conventional 10 and $25 \mathrm{fmol} / \mathrm{mg}$ as cutoff values for estrogen receptor and progesterone receptor, respectively, about $70 \%$ were estrogen receptor positive, and $49 \%$ were progesterone receptor positive.

TABLE 1. Clinicopathological and Biological Characteristics and Their Relationship with Mitotic Indices

\begin{tabular}{|c|c|c|c|c|c|}
\hline & \multirow{2}{*}{ Number of Cases (\%) } & \multicolumn{2}{|c|}{ MAI } & \multicolumn{2}{|c|}{ MI } \\
\hline & & Median (range) & $P$ & Median (range) & $P$ \\
\hline \multicolumn{6}{|l|}{ Age } \\
\hline$\leq 50$ & $116(37.9)$ & $5(0-99)$ & & $0.20(0.00-4.55)$ & \\
\hline$>50$ & $190(62.1)$ & $6(0-89)$ & 0.24 & $0.30(0.00-3.90)$ & 0.21 \\
\hline \multicolumn{6}{|l|}{ Menopausal status } \\
\hline Premenopause & $110(35.9)$ & $5(0-99)$ & & $0.23(0.00-4.55)$ & \\
\hline Postmenopause & $196(64.1)$ & $6(0-89)$ & 0.53 & $0.30(0.00-3.90)$ & 0.53 \\
\hline \multicolumn{6}{|l|}{ Type of surgery } \\
\hline Quadrantectomy + RT & $156(51.5)$ & $5(0-99)$ & & $0.20(0.00-3.50)$ & \\
\hline Mastectomy & $147(48.5)$ & $7(0-89)$ & 0.12 & $0.35(0.00-4.55)$ & 0.04 \\
\hline Missing & 3 & & & & \\
\hline \multicolumn{6}{|l|}{ Histotype } \\
\hline Ductal & $240(79.2)$ & $6(0-99)$ & & $0.35(0.00-4.55)$ & \\
\hline Other & $63(20.8)$ & $4(0-56)$ & 0.04 & $0.15(0.00-2.60)$ & 0.01 \\
\hline Missing & 3 & & & & \\
\hline \multicolumn{6}{|l|}{ Tumor size (cm) } \\
\hline$\leq 1.0$ & $42(14.6)$ & $3(0-51)$ & & $0.15(0.00-2.60)$ & \\
\hline $1.1-2.0$ & $146(50.9)$ & $6(0-74)$ & & $0.25(0.00-2.90)$ & \\
\hline$>2.0$ & $99(34.5)$ & $10(0-99)$ & 0.02 & $0.45(0.00-4.55)$ & 0.01 \\
\hline Missing & 19 & & & & \\
\hline \multicolumn{6}{|l|}{ Receptor status (fmol/mg) } \\
\hline \multicolumn{6}{|l|}{ ER } \\
\hline$\geq 10$ & $211(71.3)$ & $4(0-48)$ & & $0.20(0.00-1.85)$ & \\
\hline$<10$ & $85(28.7)$ & $15(0-99)$ & $<0.01$ & $0.70(0.00-4.55)$ & $<0.01$ \\
\hline Missing & 10 & & & & \\
\hline \multicolumn{6}{|l|}{ PgR } \\
\hline$\geq 25$ & $141(48.8)$ & $4(0-37)$ & & $0.20(0.00-1.65)$ & \\
\hline$<25$ & $148(51.2)$ & $9.5(0-99)$ & $<0.01$ & $0.43(0.00-4.55)$ & $<0.01$ \\
\hline Missing & 17 & & & & \\
\hline
\end{tabular}


All patients were closely followed and had a clinical, hematological, biochemical, and instrumental check-up at 3-month intervals for the first 2 years, every 6 months during the 3rd, 4th, and 5th years, and once a year thereafter up to the 10th year. During the 1st year, a biannual liver scan and chest radiograph were carried out; during the 2 nd year a chest radiograph was performed at the 6 th and 12th month, and a liver scan, at the 12th month. Subsequently, both examinations were carried out once a year. Furthermore, all patients underwent annual mammography and bone scan. Median follow up was 74 months (range, 3-123 mo).

\section{In Vitro Determinations}

Immediately after surgery, part of the tumor material was sent to the pathology laboratory for diagnosis, and part was frozen in liquid nitrogen and stored at $-80^{\circ} \mathrm{C}$ for estrogen receptor and progesterone receptor determinations.

\section{Mitotic Figure Counting}

Tumor samples were fixed in $10 \%$ buffered formalin, and $4-\mu \mathrm{m}$ sections from paraffinembedded blocks were stained with conventional hematoxylin-eosin.

Mitotic figures were counted in areas selected on the basis of the following criteria: (1) areas with exclusively infiltrating breast cancer, avoiding any in situ component; (2) presence of good cellularity, possibly localized at the periphery of the section in which active growth is most likely to occur and in fields with no necrosis, inflammation, or calcifications; and (3) high density of mitotic figures. Counting was carried out in 10 consecutive fields of $0.196 \mathrm{~mm}^{2}(400 \times)$. Mitotic figures were identified according to the criteria proposed by van Diest et al. (9). Only cells with clear morphological features of metaphase, anaphase, and telophase were counted, avoiding apoptotic and hyperchromatic nuclei.

Mitotic activity was calculated in two different ways: (1) as the number of mitotic figures in 10 consecutive fields (mitotic activity index; MAI) or (2) as the percentage of mitotic cells out of a total of 1000 neoplastic cells (mitotic index; MI). The first evaluation represents one of the parameters included in the grade scoring system of the modified Nottingham Tenovus method (10). Mitotic figures per 10 fields (MAI) and the percentage of mitotic figures (MI) were counted blindly by two observers (Observer $1=\mathrm{LM}$ and Observer $2=\mathrm{AMV}$ ).

In clinical analysis in which mitotic counts were analyzed as categorized variables, we used the cutoff values of $\leq 9$ (score 1), 10-19 (score 2 ), and $>19$ (score 3) for mitotic figures per 10 fields (MAI) and the tertiles of distribution for the percentage of mitotic figures (MI).

\section{Steroid Receptor Content}

Estrogen receptor and progesterone receptor were assayed by the dextran-coated charcoal method according to the European Organization for Research and Treatment of Cancer (11). Quality control procedures for hormone receptor dosage were coordinated by the Italian ad hoc committee.

\section{Statistical Methods}

To evaluate the reproducibility of the mitotic variables determined by two independent observers, the Spearman's correlation coefficient was calculated. A nonparametric ranking statistic (median test) and Spearman's correlation coefficient were used to investigate the relationship between mitotic figures per 10 fields (MAI) or the percentage of mitotic figures (MI) and other clinicopathological or biological factors in individual tumors.

With regard to clinical analyses, disease-free survival was calculated as the period from surgery until the first documented evidence of new disease manifestation in locoregional or distant sites or in the contralateral breast, or until death without evidence of disease. In addition, the occurrence of distant metastases as the first unfavorable event was analyzed separately. Overall survival was calculated as the period from surgery to death due to any cause.

The prognostic effect of mitotic figures per 10 fields (MAI) and of the percentage of mitotic figures (MI) was evaluated by considering them as continuous or categorical variables. Three-knots cubic spline regression was used to model in a flexible way the relationship between the logarithm of the hazard ratio and MAI and MI, considered as continuous variables.

Univariate analysis was performed, tracing Kaplan Meier curves (12), and comparison of survival curves was based on log-rank test (13).

To evaluate their independent prognostic relevance, MAI or MI were considered as categorical variables in a multivariate analysis using a Cox regression model that included other known prognostic variables such as estrogen receptor level, tumor size, and menopausal status (14).

All $P$ values were based on two-sided testing, and none were adjusted for the multiplicity of the performed tests. Statistical analyses were carried out by S-plus and SAS statistical software. 


\section{RESULTS}

\section{Reproducibility of Mitotic Count}

In the entire series of 306 cases, the matched-pair determinations by the two independent observers on individual tumors showed (Fig. 1) very high interobserver reproducibility for both MAI $\left(\mathrm{r}_{\mathrm{s}}=.89\right.$, $P<.001)$ and $\mathrm{MI}\left(r_{\mathrm{s}}=.83, P<.001\right)$. Consequently, for basic and clinical analyses, the mean of the variable evaluations of the two observers was used. Moreover, a high correlation was observed between MAI and MI values $\left(r_{\mathrm{s}}=.96, P<.001\right.$; Fig. 2$)$.

\section{Basic Results}

In our series of node-negative breast cancers, both mitotic variables varied greatly from tumor to tumor, ranging from 0 to 99 for MAI and from 0 to $4.55 \%$ for MI. The median values for the entire series were 6 mitotic figures per 10 fields and $0.03 \%$, respectively.

The clinical, pathological, and biological characteristics of patients and tumors are shown in Table 1 . The relation with the different features was similar for both mitotic indices. In particular, the number of mitotic figures was the same in premenopausal and postmenopausal patients as well as in younger and older women, whereas it was significantly higher in ductal than in other histologies, twice as high in larger than in smaller tumors, and about three times and twice as high in estrogen receptor- or progesterone receptor-negative than in positive steroid receptor lesions, respectively.

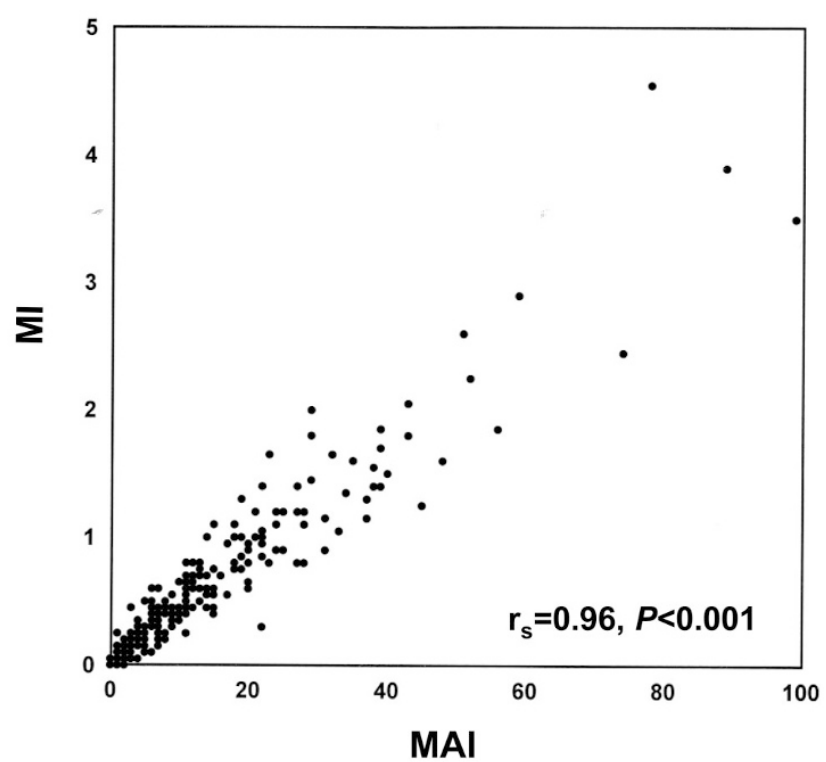

FIGURE 2. Correlation between MAI and MI.

The correlation analysis between MAI or MI and the various clinicopathological and hormonal characteristics considered as continuous variables showed a significant direct relation with tumor size $\left(r_{\mathrm{s}}=.21, P<.001\right.$ and $r_{\mathrm{s}}=.20, P<.001$, respectively) and an inverse relation with estrogen receptor $\left(r_{\mathrm{s}}=-0.29, P<.001\right.$ for both indices $)$ and progesterone receptor $\left(r_{\mathrm{s}}=-0.26, P<.001\right.$ and $\mathrm{r}_{\mathrm{s}}$ $=-0.22, P<.001$ for MAI and MI, respectively). However, it must be underlined that the correlation coefficients were always very poor.
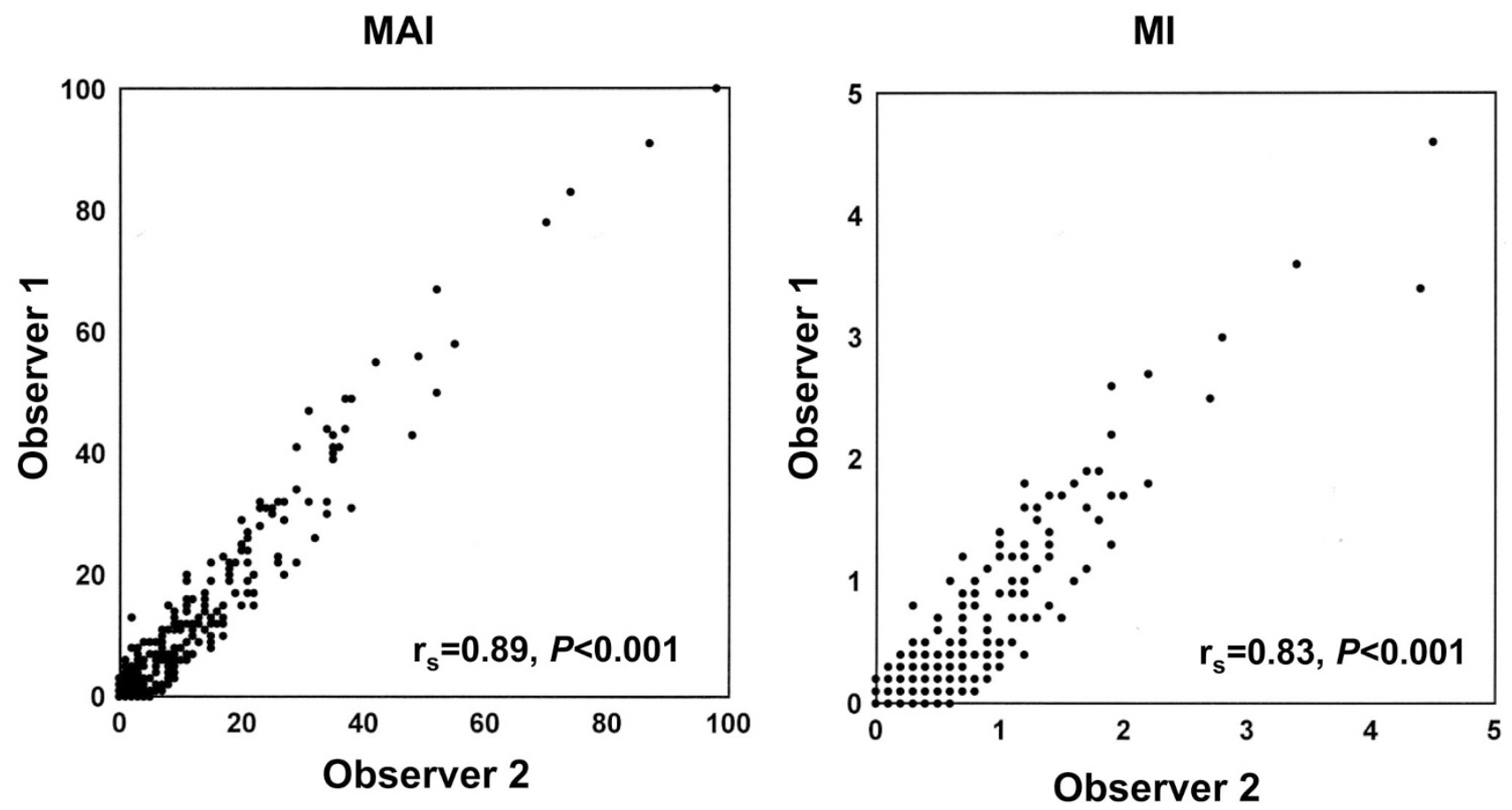

FIGURE 1. Interobserver reproducibility of mitosis determinations. 


\section{Clinical Results}

Six-year relapse-free survival for the present case series of node-negative breast cancer patients treated with locoregional therapy alone was $72 \%$, in agreement with the results obtained on large case series from clinical studies. Of the 306 patients, 69 relapsed, 19 with locoregional and 50 with distant recurrences, and 11 developed contralateral metachronous tumors. Two patients died without evidence of disease.

The mitotic values derived from each evaluation were used as continuous or categorical variables for the analysis of their clinical relevance. The application of the Cox model to the former analysis showed that neither the mitotic density nor the percentage of mitotic figures were related to disease-free survival (Fig. 3, A-B). Conversely, both MAI (Wald statistics $=12.65$ [2 df], $P=.002$ ) and MI (Wald statistics $=15.45[2 \mathrm{df}], P<.001$ ) significantly influenced the risk of death (Fig. 3, C-D).

In the latter analysis, MAI and MI were categorized in three different classes according to the cutoff values of the modified Nottingham Tenovus grading system and the tertiles of the distribution, respectively. Once again, disease-free survival was not significantly different for the three MAI or MI subgroups (Fig. 4, A-B). In particular, the highest MAI score and MI tertile subgroups showed a worse prognosis than the other two subgroups in the first years, after which the curves approximated and 6 -year disease-free survival was similar for all the subgroups. The use of other cutoffs, including the median values, led to the same results.

Different results were obtained when the relevance of mitotic indices was distinctly analyzed for the different types of early relapse. The number of locoregional and contralateral recurrences was too small to permit breakdown analyses, whereas a close correlation was observed between each mitotic variable determined at the time of diagnosis and distant metastases (Fig. 5, A-B), both in uniand multivariate analyses (likelihood ratio test [2 df] $=12.001, P<.01$ for MAI; 6.663, $P=.04$ for MI).

Furthermore, overall survival was influenced by the number of tumor mitotic figures, and the probability of overall survival at 6 years was $>95 \%$ for
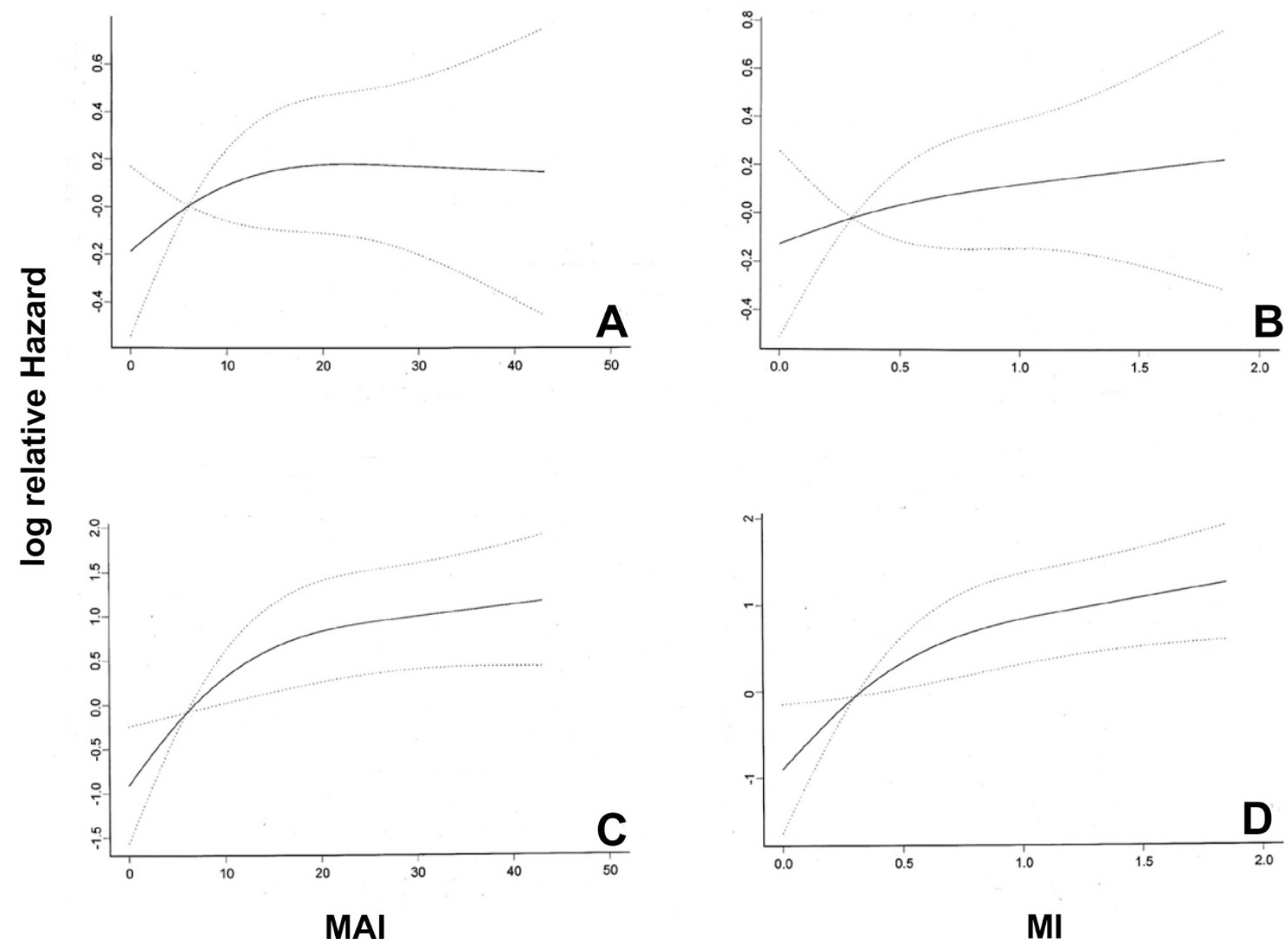

FIGURE 3. Estimated effect in univariate analysis of MAI (A) and MI (B) associated with disease-free survival (DFS) and overall survival (OS; C, D). Three-knots restricted cubic spline regression model was used. The solid line is the estimated hazard ratio (log), and the dotted lines are located at \pm 2 standard errors. 

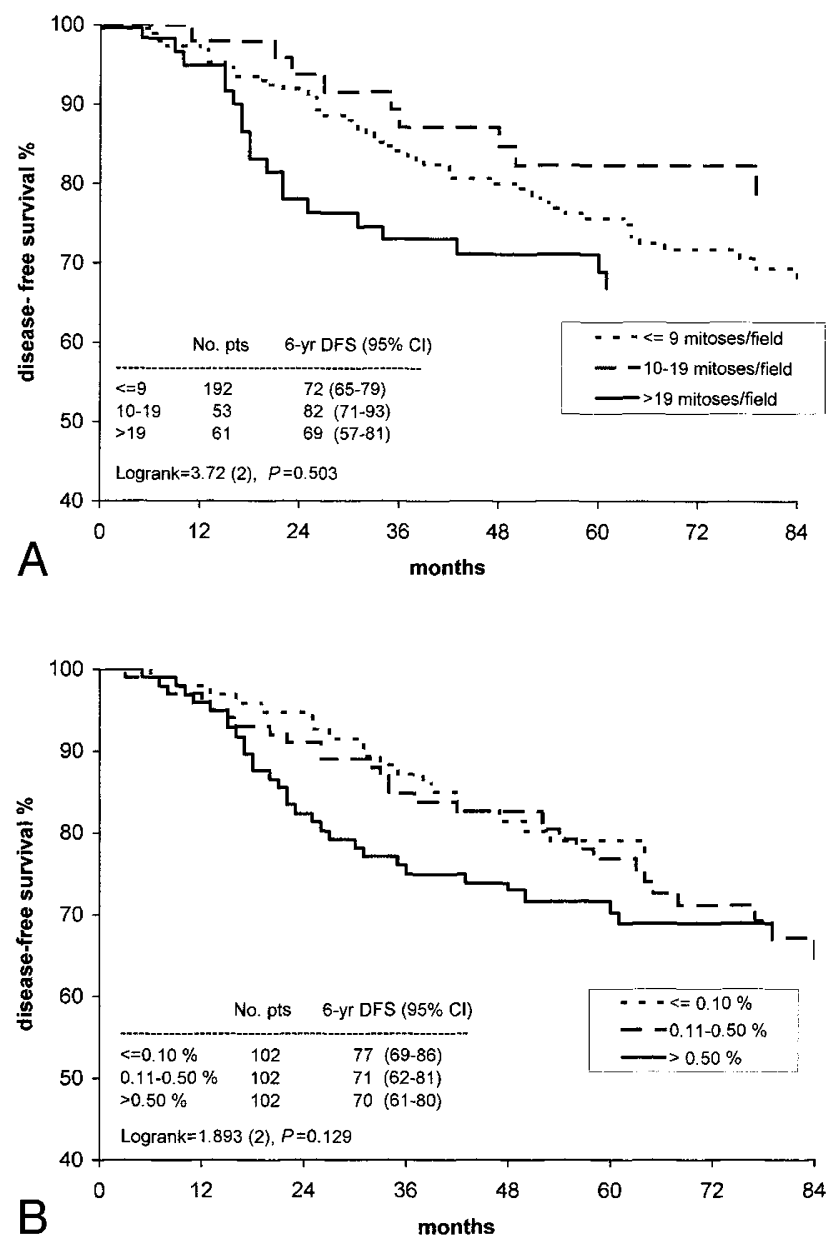

FIGURE 4. Disease-free survival as a function of MAI (A) and MI (B).

each subgroup of patients with the lowest mitotic activity, compared with about $80 \%$ for the highest subgroups (Fig. 6, A-B).

A breakdown analysis as a function of tumor size showed maximum clinical relevance of both mitotic variables, albeit more evident for MAI, in distant disease-free and overall survival in the subgroup of patients with 1- to 2-cm tumors. Conversely, only a trend was observed in the subgroup of patients with tumors of $>2 \mathrm{~cm}$ (Table 2).

Finally, in multivariate analysis using a Cox regression model containing tumor size, estrogen receptor level and menopausal status, both mitotic indices maintained their independent prognostic relevance on overall survival (likelihood ratio test $[2 \mathrm{df}]=$ 11.496, $P<.01$ for MAI; $10.939, P<.01$ for MI).

\section{DISCUSSION}

Although MI has been hypothesized as an important prognostic factor for women with infiltrating breast carcinoma, very few articles have correctly addressed the issue of the pure prognostic value of this variable. The present study provides new information with respect to that of other investigators
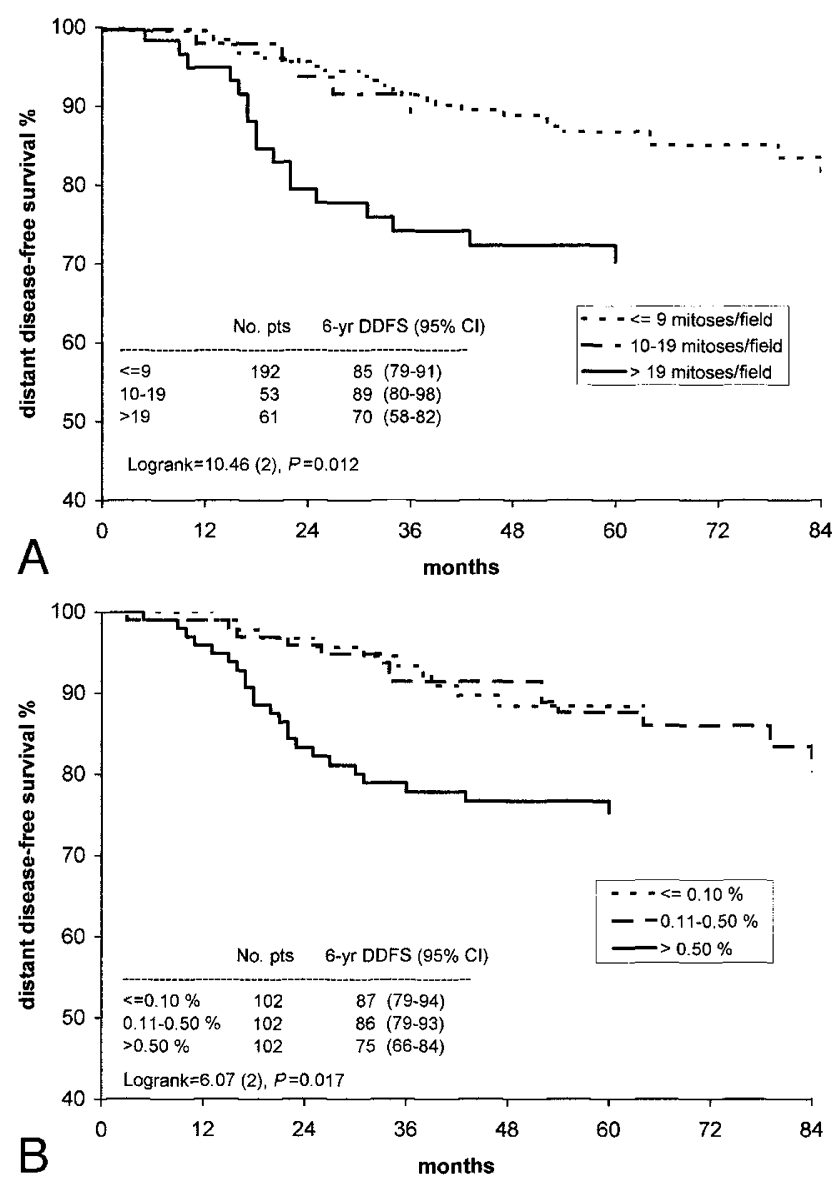

FIGURE 5. Distant disease-free survival (DDFS) as a function of MAI (A) and MI (B).

(8) in that it evaluated the clinical impact of the number of mitotic figures in tumor cell population on distant disease-free survival. To assess the pure prognostic relevance of the marker, we studied a large series of node-negative breast cancer patients treated with locoregional therapy alone, in accordance with accepted guidelines $(15,16)$.

In the light of validated and consistent results obtained with other proliferation markers, such asthymidine labeling index $(1,2)$, it is conceivable that MI could also play an important role in determining the natural history of the tumor. Nevertheless, to draw final conclusions, it is also necessary that the older morphometric measures, such as grade and mitotic activity, be tested and validated in strict conditions of intra- and interlaboratory quality control. Reproducibility among pathologists and among centers remains an issue for these markers, as for many others proposed to and used by clinicians for patient management. Sources of variability include the definition of the microscopic field area and the identification of mitotic figures, also in relation to tumor cellularity.

In the present study, we demonstrated the possibility of obtaining good interobserver reproducibility of mitotic determinations carried out blindly 

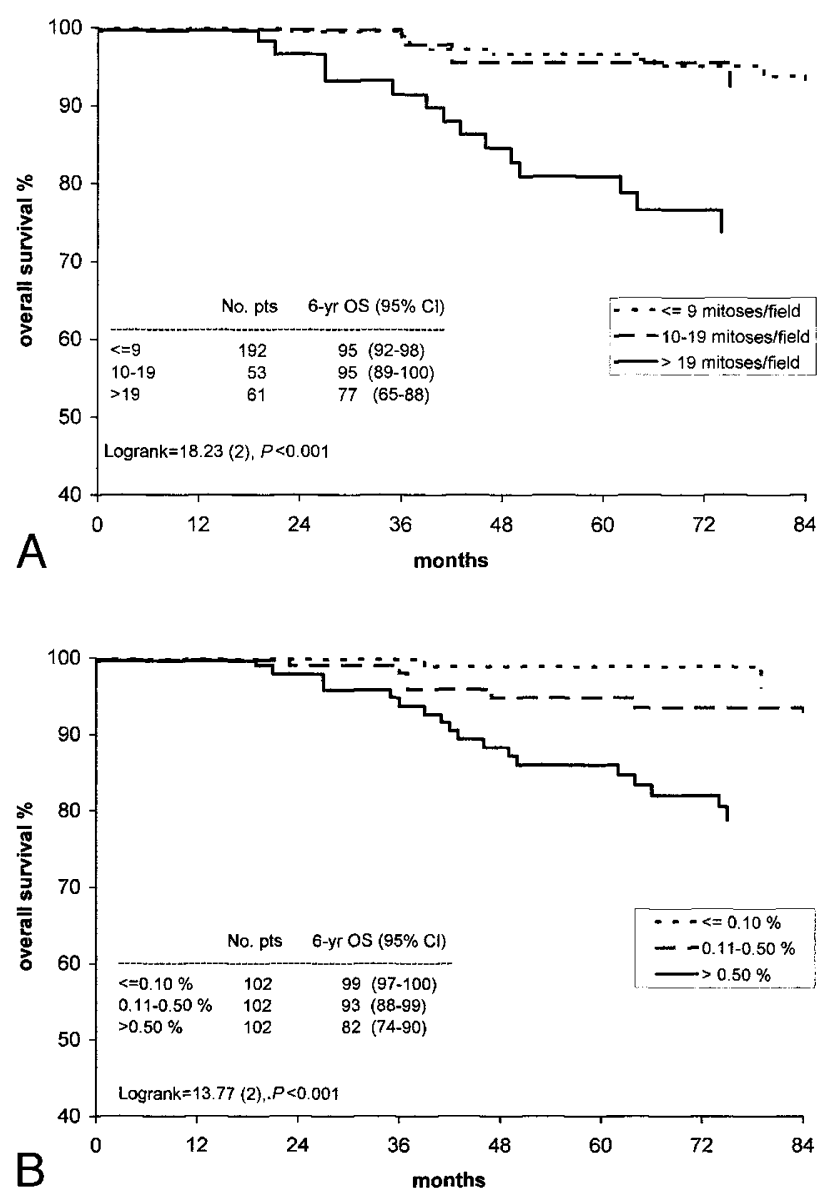

FIGURE 6. Overall survival as a function of MAI (A) and MI (B).

and observed a strong correlation between the number of mitotic figures expressed as a percentage of a total of 1000 tumor cells (MI) or per 10 fields (MAI). As a consequence, the relation between either mitotic variable and the different clinical, pathological, and biological factors was similar and consisted in higher values in ductal than in other histotypes, a direct relation with tumor size, an inverse relation with steroid receptors, and no relation with menopausal status or patient age.

One of the most critical points in defining the prognostic relevance of biomarkers measured on a continuous scale is the arbitrary choice of cutoff values (17), which could lead to the loss of part of the information and impair the comparison of results from different studies. We obtained the same basic and clinical results by analyzing the two mitotic indices as continuous or categorical variables. For the latter analysis we used the values of mitotic figures per 10 fields (MAI) according to the criteria adopted in the widely-used modified Nottingham Tenovus grading system and the tertiles of the distribution for the percentage of mitotic figures (MI).

Mitotic indices were not indicative of better or worse disease-free survival either when used as continuous or categorical variables. Conversely, both indices were able to identify subgroups of patients at different risk of developing distant metastases as the first event or of death, independently of conventional prognostic factors. More important, their prognostic impact was evident in patients with 1- to 2-cm lesions, which currently represent the majority of diagnosed tumors in Italy.

The independent role of mitotic figures on metastatic-free survival had already been observed by Mandard et al. (6) on a large series of nodenegative breast cancer patients partly treated with systemic therapy. However, Mandard and colleagues' results, as well as those from several other works $(5-7,18-25)$, do not demonstrate a pure prognostic relevance as they were obtained by studying heterogeneous case series of nodenegative and node-positive breast cancer patients who were variously treated with locoregional or systemic therapies.

Our study shows that mitotic activity is an independent prognostic variable, possibly even more

TABLE 2. 6-Year Distant Disease-Free Survival and Overall Survival as a Function of MAl and MI in Tumor Size Subgroups

\begin{tabular}{|c|c|c|c|c|c|c|}
\hline & \multicolumn{6}{|c|}{ Tumor Size } \\
\hline & \multicolumn{3}{|c|}{$1.1-2.0 \mathrm{~cm}$} & \multicolumn{3}{|c|}{$>2.0 \mathrm{~cm}$} \\
\hline & $\begin{array}{l}\text { Number of } \\
\text { Cases }\end{array}$ & $\begin{array}{c}\text { 6-yr DDFS } \\
(95 \% \mathrm{CI})\end{array}$ & $\begin{array}{c}6 \text {-yr OS } \\
(95 \% \text { CI) }\end{array}$ & $\begin{array}{l}\text { Number of } \\
\text { Cases }\end{array}$ & $\begin{array}{c}\text { 6-yr DDFS } \\
(95 \% \mathrm{CI})\end{array}$ & $\begin{array}{c}6-y r \text { OS } \\
(95 \% \text { CI) }\end{array}$ \\
\hline \multicolumn{7}{|l|}{ MAI } \\
\hline$<9$ & 94 & 85 (76-94) & 100 & 49 & $82(70-94)$ & 87 (76-98) \\
\hline $10-19$ & 24 & $95(86-100)$ & 100 & 19 & 77 (57-97) & $88(72-100)$ \\
\hline$>19$ & 28 & $62(42-82)$ & 71 (53-89) & 31 & $77(62-93)$ & $80(65-95)$ \\
\hline Logrank & & 11.49 & 20.27 & & 0.50 & 0.61 \\
\hline$P$ & & 0.003 & $<0.001$ & & 0.78 & 0.74 \\
\hline \multicolumn{7}{|l|}{ MI } \\
\hline $1^{\text {st }}$ tertile & 53 & 87 (76-98) & 100 & 28 & $86(72-100)$ & 100 \\
\hline $2^{\text {nd }}$ tertile & 49 & 84 (72-96) & 100 & 25 & $86(71-100)$ & 83 (68-98) \\
\hline $3^{\text {rd }}$ tertile & 44 & 73 (59-87) & $81(68-94)$ & 46 & $74(61-87)$ & $79(67-91)$ \\
\hline Logrank & & 4.67 & 14.00 & & 1.96 & 2.24 \\
\hline$P$ & & 0.10 & $<0.001$ & & 0.38 & 0.33 \\
\hline
\end{tabular}

DDFS, distant disease-free survival. 
important than other biomarkers known and used in a clinical setting as indicators of risk. In fact, we observed that the highest values of mitotic activity identified $20 \%$ of patients with a 6 -year cumulative risk of distant metastases and death that was 1.7 and 3.6 times higher, respectively, than that observed in patients with low-intermediate values. It must be underlined, however, that the mitotic activity was determined under strict interobserver quality control conditions.

In conclusion, these findings indicate that mitotic activity is an important imprint of tumor evolution as it exerts a determining influence on longterm clinical outcome, regardless of type of treatment, but also suggest that mitotic activity does not provide predictive information on response to systemic therapy, as, conversely, has been observed for other variables such as steroid receptors, thymidine labeling index $(3,4)$, uPA and PAI $(26,27)$ and c-erbB-2, because it is an indicator of clinical outcome in patients who have been treated with either locoregional or systemic therapy. This hypothesis requires validation in prospective clinical studies.

Acknowledgment: The authors thank Gráinne Tierney for editing the manuscript. The co-authorship of all the following trial participants is acknowledged: E. Scarpi, P. Serra, Istituto Oncologico Romagnolo, Forlì, Italy; A. Riccobon, P. Rosetti, R. Maltoni, R. Ridolfi (Department of Oncology and Diagnostics), A. Saragoni (Pathology Unit), Pierantoni Hospital, Forlì, Italy; A. Tienghi, M. Marangolo, B. Vertogen, F. Zumaglini (Department of Medical Oncology), and C. Morisi (Department of Pathology), S. Maria delle Croci Hospital, Ravenna; G. Simone, C. Salvatore (Histopathology and Cytodiagnostic Unit), and B. Stea (Clinical Experimental Oncology Laboratory), National Cancer Institute, Bari, Italy.

\section{REFERENCES}

1. Amadori D, Silvestrini R. Prognostic and predictive value of thymidine labelling index in breast cancer. Breast Cancer Res Treat 1998;51:267-81.

2. Volpi A, De Paola F, Nanni O, Granato AM, Bajorko P, Becciolini A, et al. Prognostic significance of biological markers in node negative breast cancer patients: a prospective study. Breast Cancer Res Treat 2000;63:181-92.

3. Amadori D, Nanni O, Marangolo M, Pacini P, Ravaioli A, Rossi A, et al. Disease-free survival advantage of adjuvant cyclophosphamide, methotrexate, and fluorouracil in patients with node-negative, rapidly proliferating breast cancer: a randomized multicenter study. J Clin Oncol 2000;18: 3125-34.

4. Paradiso A, Schittulli F, Cellamare G, Mangia A, Marzullo F, Lorusso V, et al. Randomized clinical trial of adjuvant fluorouracil, epirubicin and cyclophosphamide chemotherapy for patients with fast-proliferating, node-negative breast cancer. J Clin Oncol 2001;19:3929-37.
5. Clahsen PC, van de Velde CJ, Duval C, Pallud C, Mandard AM, Delobelle-Deroide A, et al. The utility of mitotic index, oestrogen receptor and Ki-67 measurements in the creation of novel prognostic indices for node-negative breast cancer. Eur J Surg Oncol 1999;25:356-63.

6. Mandard AM, Denoux Y, Herlin P, Duigou F, van de Vijver MJ, Clahsen PC, et al. Prognostic value of DNA cytometry in 281 premenopausal patients with lymph node negative breast carcinoma randomized in a control trial. Multivariate analysis with Ki-67 index, mitotic count and microvessel density. Cancer 2000;89:1748-57.

7. Clayton F. Pathologic correlates of survival in 378 lymph node-negative infiltrating ductal breast carcinomas. Cancer 1991;68:1309-17.

8. Page DL, Gray R, Allred DC, Dressler LG, Hatfield AK, Martino S, et al. Prediction of node-negative breast cancer outcome by histologic grading and S-phase analysis by flow cytometry. An Eastern Cooperative Oncology Group Study (2192). Am J Clin Oncol 2001;24:10-8.

9. van Diest PJ, Baak JPA, Matze-Cok P, Wisse-Brekelmans ECM, van Galen CM, Kurver PHJ, et al. Reproducibility of mitosis counting in 2,469 breast cancer specimens: results from the Multicenter Morphometric Mammary Carcinoma Project. Hum Pathol 1992;23:603-7.

10. Elston CW, Ellis IO. Pathological prognostic factors in breast cancer. I. The value of histological grade in breast cancer: experience from a large study with long-term follow-up. Histopathology 1991;19:403-10.

11. Piffanelli A, Pelizzola D, Giovannini G, Catozzi L, Faggioli L, Giganti M. Characterisation of laboratory working standards for quality control of immunometric and radiometric estrogen receptor assays: clinical evaluation of breast cancer biopsies. Tumori 1989;75:550-6.

12. Kaplan EL, Meier P. Non parametric estimation for incomplete observation. J Am Stat Assoc 1958;53:457-81.

13. Lawless JS. Statistical models and methods for life-time data. New York: Wiley; 1982.

14. Cox DR. Regression models and life tables. J R Stat Soc 1972;34:187-220.

15. ASCO Expert Panel. Clinical practice guidelines for the use of tumor markers in breast and colorectal cancer: report of the American Society of Clinical Oncology Expert Panel. J Clin Oncol 1996;14:2843-77.

16. Hayes DF, Bast RC, Desch CE, Fritsche H Jr, Kemeny NE, Jessup JM, et al. Tumor marker utility grading system: a framework to evaluate clinical utility of tumor markers. J Natl Cancer Inst 1996;88:1456-66.

17. Courdi A, Héry M, Chauvel P, Gioanni J, Namer M, Demard F. Prognostic value of continuous variables in breast cancer and head and neck cancer. Dependence on the cut-off level. Br J Cancer 1988;58:88-90.

18. Kronqvist P, Kuopio T, Collan Y. Morphometric grading in breast cancer: thresholds for mitotic counts. Hum Pathol 1998;29:1462-8.

19. Genestie C, Zafrani B, Asselain B, Fourquet AA, Rozan S, Validire $\mathrm{P}$, et al. Comparison of the prognostic value of Scarff-Bloom-Richardson and Nottingham histological grades in a series of 825 cases of breast cancer: major importance of the mitotic count as a component of both grading systems. Anticancer Res 1998;18:571-6.

20. Simpson JF, Gray R, Dressler LG, Cobau CD, Falkson CI, Gilchrist KW, et al. Prognostic value of histologic grade and proliferative activity in axillary node-positive breast cancer: results from the Eastern Cooperative Oncology Group Companion Study, EST 4189. J Clin Oncol 2000;18:2059-69.

21. Jannink I, van Diest PJ, Baak JPA. Comparison of the prognostic value of four methods to assess mitotic activity in 186 invasive breast cancer patients. Hum Pathol 1995; 26:1086-92. 
22. Thor AD, Liu S, Moore DH, Edgerton SM. Comparison of mitotic index, in vitro bromodeoxyuridine labeling, and MIB-1 assays to quantitate proliferation in breast cancer. J Clin Oncol 1999;17:470-7.

23. Groenendijk RP, Bult P, Tewarie L, Peer PGM, van der Sluis RF, Ruers TJM, et al. Screen-detected breast cancers have a lower mitotic activity index. Br J Cancer 2000;82:381-4.

24. Biesterfeld S, Reitmaier S. Re-evaluation of prognostic mitotic figure counting in breast cancer: results of a prospective clinical follow-up study. Anticancer Res 2001;21: 589-94.

25. de Jong JS, van Diest PJ, Baak JPA. Hot spot microvessel density and the mitotic activity index are strong additional prognostic indicators in invasive breast cancer. Histopathology 2000;36:306-12.

26. Jänicke F, Prechtl A, Thomssen C, Harbeck N, Meisner C, Untch M, et al. Randomized adjuvant chemotherapy trial in high-risk, lymph node-negative breast cancer patients identified by urokinase-type plasminogen activator and plasminogen activator inhibitor type I. J Natl Cancer Inst 2001;93: 913-20.

27. Harbeck N, Kates RE, Schmitt M. Clinical relevance of invasion factors urokinase-type plasminogen activator and plasminogen activator inhibitor type I for individualized therapy decisions in primary breast cancer is greatest when used in combination. J Clin Oncol 2002;20:1000-7. 